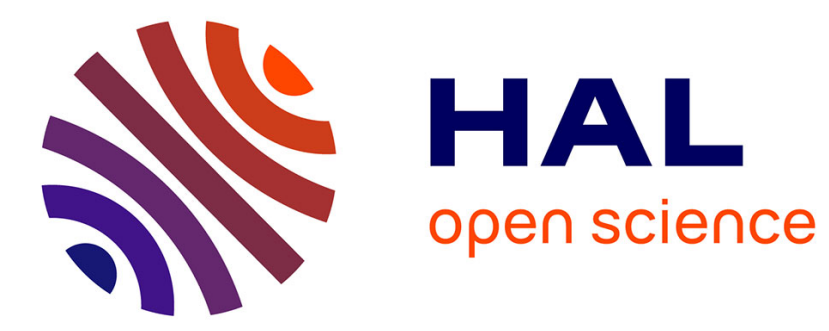

\title{
Répétitions et reformulations sur les emballages de produits courants : une quadruple illusion \\ Clara Romero
}

\section{To cite this version:}

Clara Romero. Répétitions et reformulations sur les emballages de produits courants: une quadruple illusion. Les cahiers de praxématique, 2009, 52, pp.133-158. halshs-00633918

\section{HAL Id: halshs-00633918 \\ https://shs.hal.science/halshs-00633918}

Submitted on 19 Oct 2011

HAL is a multi-disciplinary open access archive for the deposit and dissemination of scientific research documents, whether they are published or not. The documents may come from teaching and research institutions in France or abroad, or from public or private research centers.
L'archive ouverte pluridisciplinaire HAL, est destinée au dépôt et à la diffusion de documents scientifiques de niveau recherche, publiés ou non, émanant des établissements d'enseignement et de recherche français ou étrangers, des laboratoires publics ou privés. 


Titre : Répétitions et reformulations sur les emballages de produits courants : une
quadruple illusion
Auteur: Clara Romero / clara.romero @ parisdescartes.fr
Référence : (2009) Cahiers de praxématique 52, p. 133-158
Résumé:
Ayant posé la répétition comme phénomène structurant le genre publicitaire à chaque niveau
d'analyse du langage et de la communication (phonique, lexical, sémantique, intertextuel intra et
extragénérique etc.), nous étudions un corpus d'emballages de produits courants (en particulier
des cosmétiques). La reformulation, qui associe reprise et différence, y tient une place
prépondérante, associée à des fonctions particulières. Celles-ci consistent essentiellement à
donner au consommateur l'illusion que le discours qu'il lit a un contenu complexe (ceci visant à
contrer les effets délétères de la répétition), l'illusion d'être informé (fonction pseudo-
didactique), l'illusion de la garantie scientifique (fonction pseudo-scientifique), enfin, l'illusion
d'avoir le choix.
Abstract:
Repetition and reformulation on the packaging of everyday products: a fourfold illusion /
Repetition is shown to be a structural aspect of the advertising genre at all levels of analysis of
language and communication (phonic, lexical, semantic, intertextual intrageneric and
extrageneric - when compared to other texts within or outside of the advertising discourse). This
is investigated through a corpus of packaging of everyday products (in particular cosmetics).
Reformulation - which combines repetition and differentiation - is a predominant aspect of this
discourse, and is associated with specific functions. These consist essentially of giving the
consumer the illusion that the content of the discourse they are reading is complex (which
counters the wearing effect of repetition), the illusion of being informed (pseudo-didactic
function), the illusion of scientific credentials (pseudo-scientific function), and the illusion of
having a choice.

... pour une peau intensément hydratée

La Douche crème Hydratation intense lave en douceur, hydrate immédiatement et durablement les peaux sèches et déshydratées.

<Le Petit Marseillais>

\section{Point de départ : la répétition dans la publicité}

Le fait est désormais assez bien connu que la répétition irrigue (et même structure) le discours publicitaire, sous diverses formes et à chaque niveau de l'analyse du langage et de la communication. Nous avons ailleurs (Romero, 2010) détaillé cela, en montrant que, dans un même message publicitaire, ces différents niveaux de répétition se superposent ou plutôt s'imbriquent. Ont ainsi été mis au jour :

- Niveau (1), phonique: (V. Grunig, 1998). Rythmique et/ou phonémique, ce type de répétition est plus particulièrement manifeste dans les slogans, notamment sous forme de rimes (Pas d'erreur / C'est Lesieur), anti-rimes (Choisissez bien / Choisissez But), assonances (Pour toujours et surtout pour tout de suite <Dior>) et allitérations (Crunch, le chocolat qui croustille). 
- Niveau (2), d'ordre lexical : Il s'agit soit - dans un slogan - de figures ayant un effet de sens particulier (antanaclase : Prenez votre cour à cour ; Lave plus blanc que blanc, Elle est légère, légère <Lesieur, Huile de tournesol>), soit - dans une unité plus longue - de la répétition de plus de deux occurrences de morphèmes, de mots (en particulier le nom de la marque ou du produit), ou de syntagmes.

- Niveau (3), sémantique et argumentatif : C'est la répétition dans un message d'un signifié qui ne s'accompagne pas (nécessairement) de la répétition du signifiant ; par exemple au moyen de : mal, douleur, -algie dans une publicité pour un antalgique. Il semble parfois qu'une même idée soit répétée en guise d'argumentation, nous y reviendrons longuement.

- Niveau (4), textuel : Ce niveau concerne le message dans son intégralité, répété tout au long d'une campagne. Le nombre de répétitions correspond alors au nombre d'espaces publicitaires achetés par le média planning. Les messages répétés connaissent parfois des variantes, tout en restant identifiables à une série.

- Niveau (5, intertextuel intragénérique : Une répétition relève de ce niveau lorsqu'un message intègre des éléments (séquences figées, structures, ou idées) provenant d'autres messages publicitaires. Ainsi l'énoncé Ressourcez-vous est-il associé à un grand nombre de produits, de types très divers (café, maillot de bain, lessive...).

- Niveau (6), intertextuel extragénérique : Dans le même ordre d'idées, les messages peuvent aussi contenir des éléments provenant de discours non publicitaires. Il peut s'agir de séquences telles quelles (On n'attrape pas les mouches avec du vinaigre <Lise Charmel, lingerie>, Prosper, Youp'là boum! <Van Damme, Pain d'épices Prosper $>^{1}$, ou modifiées (Qui sème les fleurs récolte la tendresse <Interflora>).

- Niveau 7 , « interlocutoire $»^{2}:$ Il arrive que les récepteurs répètent eux-mêmes ce discours, au point, de temps en temps, d'en faire pénétrer des parcelles dans la langue courante (ex. le slogan Un verre ça va, deux verres ça va, trois verres bonjour les dégâts! <Prévention Routière> et ses variantes dans l'oral spontané).

- Niveau (8), générico-illocutoire: L'omniprésence de la publicité entraine la répétition de l'acte illocutoire directif qu'accomplit ce genre de discours. ${ }^{3}$

\section{Rapport entre répétition et reformulation et intérêt de la reformulation}

La notion de répétition, prise jusqu'ici dans le sens d'itération d'un élément (qui peut être d'un ordre quelconque, linguistique ou non ${ }^{4}$ ), rencontre celle de reformulation, à la fois strictement linguistique et plus complexe, puisqu'elle s'appuie sur la combinaison d'éléments dans un énoncé. Nous définissons la reformulation par la reprise, dans un énoncé reformulé, d'un ou de deux parmi les paramètres suivants de l'énoncé source : le lexique, la structure syntaxique, ou le sens général (Martinot et al., 2008). Ce sont ces paramètres repris qui constituent l'aspect répétitif qui nous avaient d'abord amenée à voir ces énoncés sous l'angle de la répétition. Mais pour qu'il y ait reformulation, il faut aussi que l'un au moins de ces trois paramètres diffère de la source. En combinant de cette manière ces trois paramètres, on obtient six types de

\footnotetext{
${ }^{1}$ Chanson originale de Maurice Chevalier, 1935.

${ }^{2}$ Pour autant que l'on puisse parler d'interlocution dans la communication de masse.

${ }^{3} \mathrm{NB}$ : Les numéros attribués à chaque niveau de répétition seront repris dans le présent article. Si leur appellation a pu changer, leur numéro correspond à celui des paragraphes dans Romero, 2010.

${ }^{4}$ Il peut s'agir d'unités infralinguistiques (traits phoniques: Du beau, du bon, Dubonnet), éventuellement de graphèmes, ou de catégories d'ordre pragmatique.
} 
reformulations (v. tableau ci-dessous, le type 7 étant un cas à part puisqu'il correspond à une répétition d'énoncé).

\begin{tabular}{|l|c|c|c|l|}
\hline Type & Lexique & Structure & Sens & \multicolumn{1}{c|}{ Exemples } \\
\hline 1 & $=$ & $=$ & $\neq$ & Le garçon regarde la fille / La fille regarde le garçon \\
\hline 2 & $\neq$ & $=$ & $\neq$ & $\begin{array}{l}\text { Les chats aiment le lait / Les chiens aiment la viande } \\
\text { Les chats aiment le lait / Les chats détestent le lait }\end{array}$ \\
\hline 3 & $=$ & $\neq$ & $\neq$ & Les chats aiment le lait / Le lait n'est pas aimé des chats \\
\hline 4 & $\neq$ & $\neq$ & $=$ & Le juge interroge le suspect / Le magistrat pose des questions au prévenu \\
\hline 5 & $\neq$ & $=$ & $=$ & Le juge interroge le suspect / Le magistrat questionne le prévenu \\
\hline 6 & $=$ & $\neq$ & $=$ & $\begin{array}{l}\text { Cette affaire concerne mon voisin / Mon voisin est concerné par cette affaire } \\
\text { Le médecin examine la patiente / Le médecin procède à l'examen de la patiente }\end{array}$ \\
\hline 7 & $=$ & $=$ & $=$ & Les chats aiment le lait / Les chats aiment le lait \\
\hline
\end{tabular}

L'application à un corpus d'emballages de produits courants de la grille d'analyse par niveaux évoquée en préambule a fait ressortir l'importance et le rôle particulier des reformulations. Cependant, si cette typologie des reformulations nous servira de point d'appui, elle s'avèrera souvent trop rigide, dans la mesure où dans bien des cas, une seule constante est pour nous pertinente. De plus, si le lexique n'est que partiellement identique, notre propos s'appuiera tantôt sur le fait qu'il l'est, tantôt sur le fait qu'il ne l'est pas. Enfin, pour ce qui est du sens, nous serons confrontée aux difficultés théoriques de la définition de la paraphrase (Fuchs, 1980), et, de la même façon, il pourra être intéressant de relever soit l'identité soit la différence de sens entre deux énoncés sémantiquement proches. Un des paramètres de la perception de l'équivalence sémantique est celui du niveau auquel on se situe (littéral ou plus global). Ainsi, si nous ferons dès le début référence au type de reformulation dont nos exemples de corpus se rapprochent, en précisant parfois le choix possible et le point de vue adopté, ces références se feront moins présentes au fur et à mesure de l'exposé, où nous nous contenterons alors d'en souligner la constante pertinente.

\section{Note sur la constitution du corpus et premières observations}

Rappelons avant d'entrer dans le vif du sujet que, si ce n'est pas là leur unique fonction, les emballages sont à considérer comme des supports publicitaires à part entière ${ }^{5}$. Ils constituent d'ailleurs, pour la plupart des produits de la grande distribution, la seule publicité existante, ou du moins la seule à laquelle de nombreux consommateurs seront exposés ; et font d'ailleurs l'objet, au sein du secteur publicitaire, d'un domaine appelé packaging. Notre corpus comprend une quarantaine d'emballages - nous en reproduisons ici douze - recueillis non systématiquement (sauf pour les déclinaisons d'un produit), dont environ une moitié de cosmétiques, l'autre moitié comprenant des produits alimentaires (sucre, yaourt, pâtes, lait en poudre...) et de la lessive. Un tel échantillon permet déjà d'apercevoir certaines régularités. Comme nous le verrons, les

\footnotetext{
5 Jusqu'à trois niveaux («couches ») d'emballage sont couramment présentés au consommateur: 1/ le conditionnement (ex. un tube, pour de la crème), 2/ l'emballage primaire (qui emballe le produit conditionné, ex. une boite, contenant le tube), 3/ l'emballage secondaire (qui emballe un lot, ex. un film plastique regroupant deux boites). Les trois peuvent être de véritables supports publicitaires. Dans cet exposé, un emballage équivaut à un message, qu'il soit composé d'une seule ou de plusieurs couches.
} 
caractéristiques du discours publicitaire sont plus affirmées pour les cosmétiques que pour les autres produits, ce qui nous a incitée à en rassembler davantage. Les textes de ces emballages sont tout d'abord plus longs que les autres. Ceci s'explique en partie par le fait que les cosmétiques n'étant pour la plupart absolument pas indispensables, le discours qui les entoure a à persuader du contraire, par exemple en donnant des arguments d'ordre technique, les positionnant alors comme des produits techniques (qui requièrent donc une notice d'utilisation, comme pour un appareil ou un médicament). Ils contiennent ensuite davantage de reformulations que les autres. En comparaison, le discours qui accompagne un pot de confiture ou un paquet de pâtes est généralement à la fois plus bref et moins reformulatoire. On y trouvera juste éventuellement, outre le nom de la marque et la liste des ingrédients, une recette de cuisine.

\section{Étude du corpus}

En reprenant les différents niveaux d'analyse (1) à (8), nous constatons que tous ne sont ni également représentés dans les emballages, ni donc également pertinents pour les décrire spécifiquement.

- Pour le niveau (1) (répétition phonique) par exemple, les emballages ne contiennent rien de plus que d'autres supports publicitaires, et d'ailleurs plutôt moins, puisque les slogans associés à une marque n’y figurent pas nécessairement.

- Nous commencerons donc par le niveau (2) (répétition simple d'ordre lexical: morphèmes, mots, syntagmes). Ce qui saute aux yeux, c'est la répétition du nom du produit (associé au nom de la marque, et parfois à d'autres mots) sur les différentes faces (et « couches ») de l'emballage. Supposée permettre la reconnaissance du produit de quelque côté qu'il soit vu (et l'on ne peut voir tous les côtés en même temps), cette répétition est au départ plus «spatiale » que «temporelle ${ }^{6}{ }^{6}$ Cependant, le produit étant maintes fois manipulé pendant sa période d'utilisation, elle aboutit quand même à faire percevoir ces mots un plus grand nombre de fois, ce qui est l'objet même de la répétition publicitaire.

- Observons à présent un exemple typique de répétition relevant d'abord du niveau (2) (reformulation de type 3 du tableau ci-dessus, à lexique constant) mais aussi, semble-t-il, du niveau (3) (sémantique, ce qui correspondrait alors au type 6 de reformulation), nous verrons pourquoi. Cet extrait de message <(1) Dash 2enl, Lessive> est constitué de trois parties : [1] un énoncé averbal ou «titre », [2] une phrase assertive, [3] une phrase impérative.

[1] Dash 2en1 (avec adoucissant naturel) Source de Fraicheur aux minéraux essentiels

[2] Dash 2en1 Source de Fraicheur aux minéraux essentiels procure à vos vêtements une propreté éclatante et une fraicheur naturelle.

[3] Ressourcez-vous avec Dash 2en1 Source de Fraicheur et retrouvez la fraicheur d'une source d'eau vive en pleine nature.

D'abord, tous les morphèmes lexicaux de [1] (sauf adoucissant) sont repris dans au moins [2] ou [3] et presque toujours dans les deux. Ensuite, [2] ainsi que [3] se reformulent elles-mêmes, avec

\footnotetext{
${ }^{6}$ Il est possible d'envisager la présence simultanée d'occurrences comme de la répétition (v. Romero, 2010, § 4) en imaginant un récepteur idéal qui, dans ce cas, lirait d'une traite tout ce qui est écrit sur une boite.
} 
en outre - pour [3] - une reformulation de type 2, à constante structurale : le préfixe re- et la marque de l'impératif $2^{\text {ème }}$ personne: re-...ez. Ainsi, du fait de la recombinaison de ces morphèmes dans des structures différentes, les trois parties ont un sens littéral différent. L'impression de répétition se trouve nettement atténuée, et le texte semble même être construit «normalement».

Pourtant, si l'on se livre à quelques calculs lexicométriques élémentaires, on constate, chose inhabituelle, que deux morphèmes lexicaux arrivent en tête des fréquences. Sur 29 morphèmesoccurrences lexicaux, 20 concernent des morphèmes-types qui apparaissent plus d'une fois, et 13 (soit près de la moitié) concernent le seul syntagme plus ou moins développé Dash 2enl source de fraicheur (aux minéraux essentiels), correspondant au nom (étendu) du produit. ${ }^{7}$ Une telle pauvreté lexicale ne peut être sans conséquence sur le sens de l'ensemble. Ce dernier semble reposer non sur l'articulation, mais sur l'évocation de quelques concepts, en plus du nom de la marque (3 occurrences): source (5 occurrences), nature (3 occurrences), fraicheur (5 occurrences), que l'on fait ensuite alterner (non régulièrement), sans avoir l'air de les marteler. L'énoncé n'est donc pas à interpréter littéralement. À un niveau plus abstrait, il apparait que ces trois phrases n'ont pas un sens tellement différent, puisque celui-ci ne fait que combiner (différemment) les mêmes éléments, utilisés pour leur pouvoir évocateur. Ce «sens » est d'ailleurs suffisamment flou, puisqu'à cela il faut ajouter l'usage de termes axiologiques (éclatant), d'impératifs, de figures (métaphore, hyperbole...), pour n'apporter aucune information vérifiable. ${ }^{8}$

Dans l'exemple suivant <(2) Régilait, Lait en poudre>, relevant clairement du niveau (3), le lexique repris est bien moins important, la reprise étant davantage sémantique (donc proche du type 4 de reformulation).

[recto] Régilait - BIO (issu de l'agriculture biologique) - lait écrémé - [logo : certifié $\mathrm{AB}$ AGRICULTURE BIOLOGIQUE] - LAIT INSTANTANE 250g = l'équivalent de 2,5 litres de lait

[verso] BIO - lait écrémé - 5 ENGAGEMENTS POUR LA QUALITE

- Engagement n ${ }^{\circ} 1$ : Qualité Bio certifiée

Régilait Bio est un lait écrémé issu de fermes pratiquant l'Agriculture Biologique, un mode de production respectueux des animaux et de l'environnement. Régilait Bio a été certifié par un organisme indépendant ECOCERT SAS F32600.

- Engagement $n^{\circ} 2$ : Respect de la nature

Les méthodes de production sont plus «douces » pour la terre grâce, notamment, à l'utilisation de fertilisants naturels.

- Engagement $n^{\circ} 3$ : Des vaches bien traitées

Les vaches vivent dans des prairies entretenues de façon écologique, sans traitements intensifs, conformément à la réglementation. Leur eau est régulièrement contrôlée et leur alimentation est essentiellement composée de fourrage frais et de céréales...

- Engagement $n^{\circ} 4$ : Un lait de qualité

Chez Régilait, les méthodes de transformation du lait liquide en poudre de lait sont simples et douces : le lait liquide est pasteurisé puis séché pour évaporer l'eau qu'il contient et ne conserver que le meilleur du lait.

- Engagement n ${ }^{\circ}$ : Respect de l'environnement

Régilait Bio est conditionné dans un étui recyclable.

\footnotetext{
${ }^{7}$ Quant aux 9 morphèmes restants (qui n'apparaissent qu'une fois), ils ne sont nullement indépendants des autres. Parmi eux, deux intensifient des éléments répétés (éclatante, pleine), deux autres répètent (au moins partiellement) le signifié de morphèmes répétés (eau vive > source, nature), un autre (adoucissant) reprend partiellement le signifiant de source, maintes fois répété.

${ }^{8}$ La publicité peut ainsi facilement se défendre d'être mensongère.
} 
[logo : certifié AB AGRICULTURE BIOLOGIQUE] Le saviez-vous ?

Le logo $\mathrm{AB}$ est une marque de Ministère de l'Agriculture. Il peut être apposé car Régilait Bio a été reconnu produit issu du mode de production biologique.

Là encore, on voit que toute l'information ou presque se trouve déjà sur le recto et dans le titre (« lait écrémé », «bio »), que le reste du texte ne fait que paraphraser en la développant. Ensuite, le même phénomène se reproduit au niveau des alinéas, dont plusieurs ne font que reformuler leur titre (reformulations de type 4 ou 6). Enfin, ce qui apparait sous forme d'une énumération en cinq points correspond en fait soit à des développements d'éléments antérieurs (les engagements 2 et 3 sont contenus dans le 1 , reprenant lui-même le concept «bio »), soit à des éléments très faiblement informatifs, voire tautologiques (car ils sont vrais de tous les produits du même type), mais visant à renchérir sur le caractère «naturel » du produit (engagements 4 et 5). Notons en outre que, s'il s'agit là de paraphrases se voulant explicatives ou définitoires (après tout, le consommateur visé ne sait pas nécessairement que le logo $\mathrm{AB}$ condense à lui seul un cahier des charges bien précis et une certification, et que la légalité de la mention bio en découle), il y a cependant lieu de penser que l'idée vague que le consommateur se faisait du «bio » avant de lire l'emballage, ne se trouvera guère précisée après sa lecture. En effet, respectueux des animaux et de l'environnement, méthodes (...) « douces », de façon écologique, sont des termes extrêmement vagues, tandis que l'alimentation (...) essentiellement composée de fourrage frais et de céréales... laisser planer le doute sur le complément ; seuls utilisation de fertilisants naturels et sans traitements intensifs viennent apporter un peu de précision. De même, que le lait soit séché pour évaporer l'eau qu'il contient ${ }^{9}$ par des méthodes simples et douces est fait pour rassurer, mais n'explicite pas en quoi celles-ci consistent!

Pour finir, plusieurs alinéas reformulent un alinéa antérieur en conservant surtout la structure (reformulation de type 2). Voici quelques parcelles constituant des constantes ${ }^{10}$ :

\section{1) Engagement $n^{\circ}$ Nombre : [(dét.) N Attribut] $\vee[$ Respect de $\mathrm{N}]$}

2) SN Copule (Adv. en -ment) [Attribut] $\vee$ [Participe passé]

L'exemple <(3) $L \& H$, Sucre> en annexe ${ }^{11}$ illustre le même phénomène de paraphrasage du titre au moyen de paraphrases faiblement explicatives. De plus, peut-on vraisemblablement ignorer que le sucre roux est un produit non raffiné, possédant un gout original, etc. (information apportée) tout en sachant ce que sont les sels minéraux et oligo-éléments, leur rôle pour l'organisme (information non apportée) ?

Le but de ces deux derniers messages, qui en disent donc trop ou pas assez, n'est par conséquent pas réellement didactique, quoique leur forme voudrait le laisser croire. On peut parler de pseudo-didacticité. En faisant semblant de répondre à de fausses questions, ils en posent de réelles, sans leur apporter de réponse. Tout comme dans les jeu-concours, où les réponses des candidats n'ont pas pour but d'apprendre quoi que ce soit à celui qui les pose, les explications

\footnotetext{
${ }^{9}$ Ce segment est une nouvelle reformulation : «évaporer l'eau qu'il contient » n'est rien d'autre qu'une périphrase de «sécher ». Or pour demanderait à sa suite une information différente de celle qui précède (le but). L'énoncé reste assez naturel car l'interprétation peut jouer sur les actants des verbes : sécher (= on fait sécher) pour évaporer (= l'eau s'évapore), et/ou sur l'aspect du procès : sécher (inchoatif) / évaporer (progressif) ou sécher (progressif) / évaporer (résultatif).

${ }^{10}$ Le signe $\vee$ signifie « ou ». De part et d'autre du $\vee$, les crochets signalent les bornes des éléments de l'alternative. Les éléments facultatifs sont entre parenthèses.

${ }^{11}$ Afin de permettre à l'exposé de progresser à un rythme supportable, certains exemples ont été annexés. On pourra s'y référer lors d'une deuxième lecture.
} 
fournies par ces emballages ne servent qu'à montrer que l'on détient un savoir. Pour le publicitaire, si elle n'est pas constitutive d'un jeu, la détention de ce savoir sert au moins d'argument dans la compétition commerciale.

Un autre message - non reproduit - du corpus ( $<L u$, Petits-beurres $>)$, fait de multiples morceaux séparés, manifeste une configuration textuelle différente. Chaque morceau reprend une partie de l'information donnée dans un autre. Cet artifice de présentation (encadrés, typographie, etc.) atténue le fait que, s'agissant au fond d'un unique message, la progression de l'information d'un morceau à l'autre s'y fait au rythme de «deux pas en avant, un pas en arrière ». Là aussi, il faut compter que certains morceaux contiennent eux-mêmes des reformulations.

Ceci étant dit, tous ces exemples illustrent bien, nous semble-t-il, un phénomène repéré par Adam \& Bonhomme (1997: 144 sqq.) dans le discours publicitaire: la «captation du mécanisme argumentatif et textuel par les topoï » :

\begin{abstract}
Normalement, (...) les topoï devraient rester à l'arrière-plan et se contenter de réguler le bon déroulement de l'argumentation, laquelle doit progresser, selon un ou plusieurs sauts inférentiels, vers une conclusion à faire admettre, elle-même étant peu assurée au départ. Or souvent, loin de constituer une impulsion première appuyant un développement argumentatif, une partie des topoï publicitaires deviennent la cible de l'argumentation, celle-ci s'organisant autour d'eux dans une dynamique centripète. Au lieu que les topoï soutiennent un dépli du texte, comme ils le font habituellement, le texte se replie sur les topoï.
\end{abstract}

À titre contrastif, pour <(1) Dash 2en1, Lessive>, une argumentation qui progresserait normalement (et sans surrépétition lexicale) donnerait :

Prémisse 1 : Vous en avez assez que votre linge qui sort de la machine ne soit pas assez propre et ne sente pas assez bon?

Prémisse 2 (topos) : Heureusement, il y a Dash 2en1 Source de Fraicheur aux minéraux essentiels, la seule lessive qui procure à vos vêtements une propreté éclatante.

Conclusion : Courez vite l'acheter!

Les emballages de cosmétiques possèdent ces caractéristiques : reformulations à constante lexicale avec un fort taux de répétition, reformulations à constante sémantique (paraphrases) du topos, d'où faible informativité, «argumentation » circulaire. Mais tout cela prend pour eux une forme particulière, celle d'un discours pseudo-scientifique. Avant de montrer ceci, rappelons d'abord les arguments de Martinot (1997), issus de l'analyse d'un corpus de brochures et de notices pour des produits de beauté, à l'appui de cette pseudo-scientificité :

- Garantie explicite de la science : scientifiquement prouvé,

- Référence à la science médicale : issu de la recherche dermatologique, isomorphie des notices avec celles de médicaments (de par les différentes rubriques),

- Ambiguïté du mot soin («attention », mais aussi « acte thérapeutique »),

- Lexique pseudo-scientifique : complexe bio-hydratant HLP,

- Définitions qui sont de simples analyses linguistiques (reformulations à constante sémantique et lexicale): hypoallergénique: formulé pour minimiser les risques d'allergie.

À ces caractéristiques, il faut donc ajouter les reformulations pseudo-argumentatives imitant la structure du raisonnement (appliqué aux sciences expérimentales). En effet, tandis que dans ce domaine, un article a typiquement la structure suivante : 
1/ Hypothèse : On pose P. On veut savoir si $\mathrm{P}$ est vrai. On veut montrer $\mathrm{P}$.

2/ Protocole : On réalise une expérience visant à valider ou à invalider P. On s'y prend d'une certaine façon.

3/ Conclusion : On observe que $\mathrm{P}$ se vérifie ou pas. On en conclut que $\mathrm{P}$ est vrai ou pas.

l'exemple ci-dessous <(4) Timotei, Shampoing> en présente un calque nettement observable, sous une forme très ramassée :

\section{1/ Timotei, la recette de la nature pour les cheveux normaux - Nouvelle formule brillance}

2/ Timotei a découvert au sein de la nature des herbes sauvages spécifiques qui ont la vertu de donner de la brillance aux cheveux. Il en a sélectionné des extraits essentiels très purs et très riches pour les associer à une nouvelle formule brillance.

3/ Le résultat : la meilleure formule Timotei pour des cheveux pleins de santé, éclatants de brillance et de beauté, naturellement.

Au contraire de ce qui constitue un véritable raisonnement (lequel se prévaut ici en outre de la caution scientifique), $2 /$ et $3 /$ ne sont que des reformulations à constante au moins lexicale (type 3), mais aussi largement sémantique (type 6) de 1/. Ici, la partie $2 /$ concerne le protocole expérimental en amont de la fabrication du produit, et partage avec le discours de vulgarisation scientifique cet effet de "mise en scène » de la science en action. ${ }^{12}$ Il arrive que le «protocole expérimental » concerne ce qui se passe en aval de la fabrication et de l'achat, et ce, de façon minimaliste: "Lavez-vous la tête (de préférence tous les jours) avec ce shampoing ». Les exemples annexés <(5) Miss Den, Dissolvant> et <(6) Dop, Shampoing> offrent des illustrations moins «pures » de ce schéma.

Cette structure auto-reformulatoire est encore manifeste dans l'exemple <(7) Neutrogena, Crème visage>, moins condensé, mais plus complexe, puisque celle-ci se répète à plusieurs niveaux.

\section{Neutrogena Advanced Solutions}

1/ Soin hydratant lissant Advanced Solutions de Neutrogena ${ }^{\circledR}$ pour une eau hydratée, lissée et lumineuse.

3/ Bénéfices

- Action hydratante : la peau est intensément hydratée et apaisée. Elle reste douce et confortable pendant $24 \mathrm{~h}$.

- Action lissante : la texture de la peau est visiblement unifiée. La peau est plus souple, plus douce, plus lisse, avec moins de ridules visibles.

- Action sur l'éclat : le teint terne est instantanément transformé et plus lumineux.

2/ Innovation technologique

Développée avec des dermatologues, sa formule enrichie en soja naturel contient un complexe unique alliant ingrédients hydratants et nourrissants et des micro-pigments révélateurs d'éclat pour une peau hydratée, lissée et éclatante.

Non comédogène. Adapté à tout type de peau.

Texture

Sa formule veloutée et fraiche est rapidement absorbée par la peau.

2/ Conseils d'utilisation

Appliquez tous les jours sur un visage propre. Vous pouvez utiliser le masque Peeling Advanced Solutions de Neutrogena ${ }^{\circledR}$ pour une peau instantanément retexturée et lissée.

D'abord on note que l'ordre $2 /$ et $3 /$ est inversé. Les multiples petits morceaux dont est fait ce message ne sont pas tous aisément rapportables à l'une des trois parties, et pour cause. Non seulement $2 /$ et $3 /$ reformulent $1 /$, mais $3 /$ présente également des reformulations, et ce, à plus

\footnotetext{
${ }^{12}$ Authier (1982) a repéré cette mise en scène avec effets narratifs d'attente, d'accélération, de suspense.
} 
d'un niveau. En effet, à la rubrique «bénéfices », il y a d'une part, pour chacun des trois points, ce qui figure de part et d'autre des deux points (sémantiquement équivalent, ce qui est à droite étant plus reformulatoire qu'explicatif, avec des constantes lexicales), mais d'autre part, les trois points sont-ils si différents entre eux ? Ils présentent eux aussi de nettes constantes structurales, mais aussi lexicales et sémantiques (en particulier les deux premiers, pour le sens). Quant à la partie 2/, les deux modes en sont ici présents : l'étape en amont (rubrique «Innovation technologique »), et l'étape en aval («Conseils d'utilisation »). Dans cette dernière rubrique, on en profite pour faire référence à un autre produit de la gamme, le masque «Peeling Advanced Solutions », dont nous n'avons pas recueilli l'emballage, mais on peut déjà deviner que celui-ci offre, en substance, les mêmes propriétés que la crème (là encore, constante structurale, lexicale et sémantique (pour une peau instantanément retexturée et lissée).

\section{Bilan provisoire}

Ainsi, que le message soit court ou long, les emballages de cosmétiques étudiés montrent une structure semblable, faite de reformulations à plusieurs niveaux. Au premier niveau, celles-ci garnissent la structure d'un pseudo-raisonnement, conférant au discours un caractère pseudoscientifique que d'autres éléments corroborent (Martinot, 1997). Plus généralement, sur les emballages de produits courants, on trouve de nombreuses reformulations, qui au lieu d'être explicatives (ce que les deux points, les c'est-à-dire laissent espérer) semblent trouver leur raison d'être dans le témoignage qu'elles constituent de l'autorité de l'émetteur (qui, lui, sait et connait les termes «savants » tels que agriculture biologique ou hypoallergénique ${ }^{13}$ ). Plus généralement encore, le taux de répétition lexicale observé, extrêmement élevé, associé à la banalité du propos (qu'on retrouvera d'un produit à l'autre, v. niveau (5), aboutit à un discours très faiblement informatif. C'est ailleurs, à l'évidence, que réside donc l'intérêt des messages : dans une sorte de bercement langagier. Le niveau d'analyse suivant montrera de quel type d'illusions ce bercement est le support idéal.

- Pour ce qui est du niveau (4), s'il s'agit de répétition textuelle totale (ou répétition stricte du message), il n'y a guère à dire au sujet des emballages. Leur répétition spatiale sur les rayonnages des supermarchés est néanmoins loin d'être négligeable pour le vendeur, et l'on sait l'importance que revêt la disposition des produits sur le lieu de vente. La quantité de produits visibles en est l'un des paramètres, et pour qu'un produit attire davantage l'attention, on aura intérêt à en disposer autant que possible à la vue du consommateur, quelle que soit la quantité de produit réellement disponible. Ci-dessous (le schéma représente en perspective cavalière un rayonnage vu de face), le consommateur voit l'emballage du produit $\mathrm{A}$ en vingt exemplaires et celui de B en dix seulement, même si le rayonnage contient davantage de produit B.

\footnotetext{
${ }^{13}$ Ceci est particulièrement net lorsque des néologismes, plus ou moins opaques, ne sont pas explicités (Par-Elastyl), ou le sont en des termes tout aussi opaques pour le consommateur moyen (Complexe Pronutris : Oméga 6 issus de la Bourrache, Antioxydants du Thé Vert, Vitamine E et Probiotiques exclusifs (v. ci-dessous <(12) Essensis, Dessert lacté>).
} 


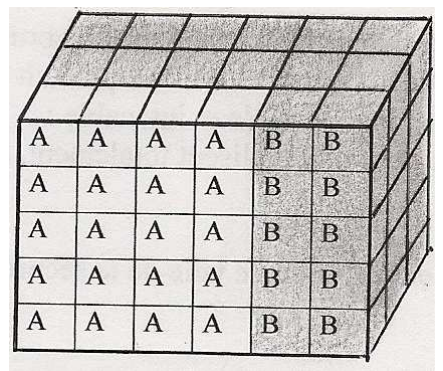

Les emballages jouent encore pleinement leur rôle publicitaire lorsqu'ils sont vus n'importe où en dehors des rayonnages. Le transport, la manipulation du produit pendant sa durée de vie ou même réduit à l'état de déchet, sont autant d'occasions répétées pour quiconque de voir encore le nom du produit. On pense également aux sacs imprimés aux couleurs d'une marque, surtout s'ils sont réutilisés, tout comme les emballages rigides (coffrets, boites en métal...) ou collectionnables, moins vite jetables.

En revanche, ce que nous avions appelé « répétition avec variante » (dans Romero, 2010), et que nous envisageons ici en termes de reformulation, trouve un développement nouveau dans les emballages de produits (cosmétiques) d'une même gamme. Plus précisément, ce sont les différentes «déclinaisons » d'un même produit en plusieurs «parfums » que nous mettrons en regard.

Une première série <(8) Casino, Douche>, que - pour la clarté de l'exposé - nous avons choisie la plus simple, permet de faire ressortir, pour les parfums (1) à (4), la trame lexico-syntaxique suivante. (Nous laissons de côté les parfums (5) et (6), qui s'écartent quelque peu de cette trame.) 14

Lait de douche $\left[\mathbf{N}_{\mathbf{1}} \mathbf{A} \mathbf{d} \mathbf{j}_{\mathbf{1}}\right] \vee\left[\mathbf{A d j}_{\mathbf{1}} \mathbf{N}_{\mathbf{1}}\right]$

Avec le lait de douche Casino $\left[\mathbf{N}_{\mathbf{1}} \mathbf{A} \mathbf{A} \mathbf{j}_{\mathbf{1}}\right] \vee\left[\mathbf{A d j}_{\mathbf{1}} \mathbf{N}_{\mathbf{1}}\right]$, transformez votre douche en un véritable moment $\left[\mathbf{A d j}_{\mathbf{2}}\right] \vee\left[\mathrm{de} \mathbf{N}_{\mathbf{2}}\left(\mathbf{A d j} \mathbf{j}_{3}\right)\right.$ ]. De $\mathrm{pH}$ neutre pour la peau, sa formule enrichie en provitamine B5 (et en extrait de $\mathbf{N}_{1}$ ) laisse votre peau souple, douce et hydratée. Sa mousse onctueuse et sa texture crémeuse vous procurent une agréable sensation de bien-être en enveloppant votre corps d'un parfum $\mathbf{A d j}_{\mathbf{4}}$ et $\mathbf{A d j}_{\mathbf{5}}$.

On pourrait donc d'abord penser qu'il s'agit d'une reformulation de type 2 (à constante structurelle). Cependant, comme il est impossible de dire quel message reformule quel autre, puisqu'ils existent simultanément, nous admettrons que tous reformulent un message plus abstrait, dont le sens (plus abstrait aussi) est le même pour tous. On peut donc parler de reformulation de type 5 (à constante structurelle et sémantique). D'ailleurs, au parallélisme structurel, il faut ajouter les éléments suivants :

- Les Adj. ou N. portant le même indice peuvent être identiques pour plusieurs parfums. Ainsi gourmand apparait en $\operatorname{Adj}_{5}$ pour les parfums (1), (3) et (4), plaisir en $\mathrm{N}_{2}$ pour les parfums (2) et (4).

- Des adjectifs portant des indices différents peuvent aussi être identiques : gourmand apparait aussi en $\mathrm{Adj}_{2}$ pour le parfum (1) et en $\mathrm{Adj}_{1}$ pour le parfum (4).

\footnotetext{
${ }^{14}$ Les textes complets (1) à (6) figurent en annexe. On trouvera également en annexe une deuxième série simple $<(11)$ Super Croix, Lessive>, et une troisième, au schéma plus complexe, avec plus de variables <(9) Petit Marseillais, Douche>.
} 
- Des Adj. ou N différents peuvent être synonymes entre eux : suave (2) et (4) vs doux (3) (et (5)).

En fin de compte, l'association du texte à trous ci-dessus et du schéma suivant ${ }^{15}$ permet de générer les textes (1) à (4).

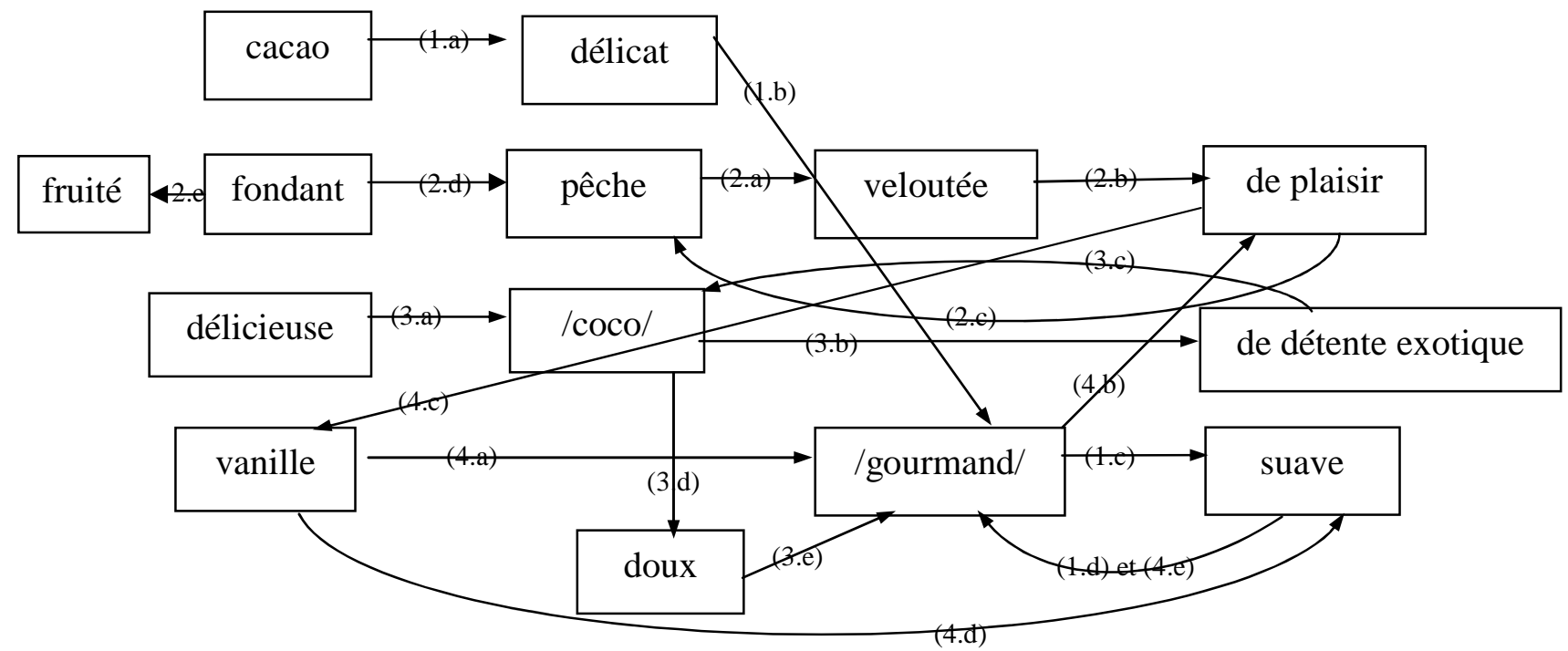

(On peut donc facilement concevoir un générateur de textes de ce type, à partir d'un texte à $n$ trous et de $n$ paradigmes.)

Tout ceci est bien (et plus amplement) illustré dans la série <(9) Petit Marseillais, Douche> (textes en annexes). Pour en revenir au sens, si les textes présentent de larges parties communes, les éléments qui varient, eux, sont censés refléter les qualités différentes des différents parfums. Or on voit que ceux-ci ne changent bien souvent que de place (nourrissant, hydratant), et qu'en outre ils ne sont pas toujours sémantiquement très différents (parfum raffiné vs délicat). Ainsi la variété des messages qui accompagne les différents parfums est-elle trompeuse. Donnant l'illusion du choix, elle ne fait que simuler la variété. Identiques (ou différents) sans l'être vraiment, associant donc identification du produit (par rapport à d'autres) et choix proposé (à l'intérieur de la gamme), les textes se trouvent dans un rapport d'iconicité d'une part avec les flacons (identiques sauf pour la couleur), d'autre part avec leur contenu :

$$
\frac{\text { texte } 1}{\text { texte } 2}=\frac{\text { flacon } 1}{\text { flacon } 2}=\frac{\text { contenu } 1}{\text { contenu } 2}
$$

La reformulation permet au surplus d'introduire l'indispensable «nouveauté » des produits (nouveau parfum, nouvelle formule...) tout en rassurant le consommateur, qui pourrait se sentir perdu ou méfiant face à quelque chose d'inconnu.

\footnotetext{
${ }^{15}$ Les éléments qui varient apparaissent encadrés et les éléments constants sont remplacés par des flèches. À chaque parfum correspond un chiffre, et l'ordre du parcours suit celui des lettres.
} 
- Au niveau (5) (intertextuel intragénérique) il s'agit de comparer de la même manière les emballages de produits différents. Commençons par des produits du même type (ex. shampoings), mais de marques différentes. Au niveau du message, et non plus de chaque phrase, on peut à nouveau voir une constante sémantique, et un lexique récurrent (transformer, doux, texture, hydrat-) tandis que la structure des phrases change (reformulations de type 6), bien que l'on puisse encore repérer des bribes communes, comme les impératifs ou l'abondance d'adjectifs qualificatifs. Pour mieux s'en rendre compte, on peut comparer $\langle(10)$ Nivea, Douche $>$ (cidessous) aux exemples déjà cités <(8) Casino, Douche> et <(9) Petit Marseillais, Douche>.

\begin{abstract}
Nivea Natural Oil - Huile de douche nourrissante
Faites l'expérience d'un soin riche et nourrissant sous la douche. Laissez son doux parfum cajoler vos sens, pendant que la texture huile de douche se transforme en une mousse soyeuse. Pour une peau agréablement douce tout au long de la journée. Ressourcez-vous chaque jour sous votre douche. Avec 55\% d'huiles nourrissantes naturelles. Adaptée aux peaux sèches.
\end{abstract}

Il semble donc que le discours entourant les produits cosmétiques soit assez homogène, quelle que soit la marque.

Les phénomènes que nous venons d'observer se retrouvent au niveau sémiologique. On a vu que le même produit, aux parfums différents, est conditionné dans des emballages identiques (seule la couleur change). Les produits de marques différentes mais d'un même type, eux, sont conditionnés dans des emballages présentant des constantes (paramètres de la couleur et de la forme), sans être identiques (ni pour la couleur, ni pour la forme). Cela contribue donc à la fois à l'identification du type de produit et à sa différentiation d'avec les produits concurrents.

En comparant pareillement des produits de types différents, nous constatons à nouveau des reformulations. Ce que nous avons dit du discours sur les cosmétiques et qui en fait un discours pseudo-scientifique se retrouve pour d'autres produits qui ne sont pas des cosmétiques, mais qui se positionnent explicitement comme tels (lessive, dessert lacté ${ }^{16}$ ) :

- Au niveau sémiologique, l'emballage (voire le produit lui-même) emprunte aux cosmétiques des paramètres de forme, de couleur, de texture.

- Au niveau linguistique, on observe :

* Vocabulaire pseudo-scientifique : l'emballage de <(1) Dash 2enl, Lessive> contient, en marge de l'extrait déjà cité, les mots (accompagnés d'illustrations) nutritonic (une écorce d'agrume) et dermadouceur (une plume de duvet), non définis, mais qui font allusion au caractère «nourrissant » des crèmes cosmétiques et à la «douceur » de la peau qu'elles promettent.

* Comme <(9) Le Petit Marseillais, Douche>, <(11) Super Croix, Lessive> (en annexe) est un produit qui contient des ingrédients «authentiques » ${ }^{17}$ aux propriétés «secrètes », et qui se décline en plusieurs parfums. Il s'agit d'ailleurs explicitement des mêmes que pour les cosmétiques (lait d'amande, fleur d'oranger, ${ }^{18} \ldots$...

\footnotetext{
${ }^{16}$ Dans ce dernier cas, la tendance exploite l'idée que «la beauté vient en mangeant», et porte le nom de cosmétofood (autres termes trouvés : cosméfood, dermonutrition, alimentation cosmétique).

${ }^{17}$ Une authenticité dite, que viennent contredire le caractère stéréotypé (industriel, en quelque sorte) du discours, ainsi que la mention en petits caractères : parfum de synthèse <(11) Super Croix, Lessive>.

${ }^{18} \mathrm{Ou}$ lait d'aloe. Aloe semble également être un nom pseudo-scientifique, puisqu'il correspond à un genre (en français aloès) et non à une espèce (par exemple aloe vera, utilisé en cosmétique).
} 
* Enfin, la structure du message peut correspondre davantage à ce que l'on trouve habituellement sur les cosmétiques, qu'à ce que l'on trouve sur les produits alimentaires, comme dans cet exemple <(12) Essensis, Dessert lacté> :

Essensis nourrit votre peau de l'intérieur

- Pourquoi avons-nous créé Essensis au PRONUTRIS ?

La santé et la beauté de votre peau dépendent aussi de votre alimentation, qui doit vous apporter tous les nutriments essentiels à votre peau, en particulier pour assurer son bon renouvellement cellulaire : votre peau se renouvelle sans cesse, et totalement en 6 semaines.

- Comment agit Essensis ?

Danone a créé Essensis pour nourrir votre peau de l'intérieur.

Grâce à son complexe exclusif PRONUTRIS qui associe Oméga 6, antioxydants et probiotiques exclusifs, Essensis apporte à votre peau les nutriments essentiels dont elle a besoin, là où les nouvelles cellules de votre peau se forment.

- En quoi Essensis est-il différent d'une crème ?

Jour après jour Essensis agit de l'intérieur sur la peau de tout votre corps.

- Essensis est-il fait pour vous ?

Oui, Essensis convient à toutes les femmes : c'est un produit laitier à $2 \%$ de mat. gr. qui contribue à la qualité de votre peau.

- Pour quels bienfaits ?

Consommé chaque jour, Essensis nourrit votre peau de l'intérieur pour une peau plus saine et donc plus belle.

Efficacité prouvée par Etude clinique

[illustration montrant des cellules en train de se renouveler sous l'action du PRONUTRIS]

Sans être des paraphrases exactes, ce qui se présente comme les réponses à différentes questions d'un consommateur critique recèle une constante sémantique (Essensis nourrit et embellit la peau de l'intérieur), le lexique n'étant pas en reste (/nourrir/, lessentiel/, /peau/, /intérieur/...) (reformulation de type 6). Grâce à cette présentation, et bien que la réponse aux questions soit donc grosso modo toujours la même, il peut sembler au consommateur qu'il a obtenu une information étoffée.

- Au niveau (6) (intertextuel extragénérique), les reformulations présentes dans le discours publicitaire en général sont observables sur les emballages au niveau du message entier. Nous avons vu ci-dessus que certains messages, qui contenaient des reformulations, empruntaient la structure de textes scientifiques ou à connotation telle. Or cet emprunt, maintenant le paramètre structurel, et jouant sur les autres paramètres, est de nature reformulatoire.

- Au niveau (7) («interlocutoire »), il n'y a rien de remarquable. Contrairement aux spots radiophoniques ou télévisés, les emballages, rarement lus à haute voix, ne sont pas la source de répétitions ou de reformulations par les récepteurs. 
- Au niveau (8) (générico-illocutoire), on note que les emballages tiennent leur place dans le «matraquage» publicitaire qui consiste à faire que le message soit reçu autant de fois que possible, par quelque voie que ce soit. Ils reprennent au moyen de répétitions et de reformulations, que nous n'avons pas jugé nécessaire d'envisager ici, le discours des affiches, spots télé ou radio.

D'autre part, les emballages sont à eux seuls (en tant que publicité) omniprésents dans l'environnement visuel quotidien. Ainsi, au cours d'une promenade au supermarché, on compte autant de réceptions de l'acte illocutoire réalisable par l'énoncé abstrait «Achetez $\mathrm{N}_{1}$ », «Achetez $\mathrm{N}_{2}$ » que l'on perçoit d'emballages. Ce message persiste une fois les produits achetés, et tant qu'ils sont visibles.

\section{Conclusion : récapitulation du rôle des reformulations}

Résumons pour terminer le rôle particulier des reformulations sur les emballages ${ }^{19}$, mis au jour ici : quatre illusions, parfois inextricables.

1) L'illusion du sens, qui consiste à simuler un contenu complexe, afin d'éviter les effets délétères de la répétition (manque d'attention, agacement) : <(1) Dash 2enl, Lessive〉.

2) L'illusion de l'information, notamment due à une pseudo-didacticité : <(2) Régilait, Lait en poudre>, <(3) $L \& H$, Sucre>, <(12) Essensis, Dessert lacté>.

3) L'illusion de la science, par un discours pseudo-scientifique : $<(4)$ Timotei, Shampoing $>,<(5)$ Miss Den, Dissolvant>, <(6) Dop, Shampoing>, <(7) Neutrogena, Crème visage>.

4) L'illusion du choix, entre des produits en fait très peu différents, soit de la même marque, soit de marques différentes : <(8) Casino, Douche>, <(9) Le Petit Marseillais, Douche>, <(10) Nivea, Douche>, <(11) Super Croix, Lessive>.

\section{REFERENCES CITEES}

Adam J.-M., Bonhomme M. 1997, L'Argumentation publicitaire, Paris, Nathan, 145.

Autier J. $\quad$ 1982, «La mise en scène de la communication dans les discours de vulgarisation scientifique », Langue française 53, 34-47.

Fuchs C. $\quad$ 1982, La Paraphrase, Paris, PUF.

Grunig B.-N. 1998, Les Mots de la publicité, Paris CNRS-Éditions.

Martinot C. 1997, «La langue de spécialité et le discours scientifique », in Piqué J., AndreuBesó J.-V., Cuéllar M. (éds), Nau Llibres, Valencia.

Martinot C., Gerolimich S., Paprocka-Piotrowska U., Sowa M.

2008, «Reformuler pour acquérir sa langue maternelle ? Investigation auprès d'enfants français, italiens et polonais de 6, 8 et 10 ans », in Schuwer M, Le Bot M.-C., Richard E. (éds.) : Pragmatique de la reformulation, Presses Universitaires de Rennes, 221-240.

Romero C. 2010, «La répétition dans le discours publicitaire », in Pifarré A.-F. et RutiglianoDaspet S. (éds), Re-répéter - répétitions, actes des Journées d'études doctorales (16-18 avril 2007), Presses Universitaires de Savoie.

\footnotetext{
${ }^{19}$ Celui des répétitions a été souligné ici-même en cours de route, évoqué dans Romero (2010), et fera l'objet d'un autre article (en préparation) avec un point de vue psycholinguistique.
} 


\section{ANNEXES $=$ corpus cité}

$<(3) L \& H$, Sucre>

$\mathbf{L} \& \mathbf{H}$

Sucre roux de canne en poudre biologique

Ce sucre roux de canne biologique a été obtenu à partir de cannes à sucre cultivées au Brésil selon le mode de production biologique, c'est-à-dire sans engrais chimique, ni pesticide de synthèse.

Le sucre roux sélectionné par la société LOIRET \& HAENTJENS est constamment contrôlé par la société de certification indépendante ECOCERT.

Ce produit garanti pure canne et non raffiné conserve toute sa saveur son arôme et ses qualités nutritionnelles (présence de sels minéraux et oligo-éléments).

Utilisation : pâtisserie, yaourts, crèmes, crêpes, salades de fruits, fromages blancs, boissons (jus de fruits, cocktail).

\section{$<(5)$ Miss Den, Dissolvant >}

\section{1/ Miss Den - Dissolvant doux}

Le dissolvant doux Miss Den élimine en douceur le vernis.

2/ Enrichie en glycérine et en huile de ricin, sa formule a été conçue pour protéger et nourrir l'ongle.

3/ Résultat : vos ongles sont parfaitement nets.

\section{$<(6)$ Dop, Shampoing>}

\section{1/ DOP, shampooing très doux protecteur à l'extrait naturel de vanille}

Le shampooing DOP lave vos cheveux tout en douceur, les rend incroyablement propres et faciles à coiffer.

3/ Touchez vos cheveux : ils sont plus doux, plus beaux et tellement brillants !

2/ Sa formule au $\mathrm{pH}$ doux convient à tous les types de cheveux : elle est idéale pour toute la famille. Vous pouvez l'utiliser aussi souvent que vous le souhaitez. Sa mousse onctueuse et son parfum agréable font du shampooing un véritable moment de plaisir.

2/ Les vertus de la vanille : la vanille est particulièrement appréciée pour sa douceur. La formule DOP à l'extrait naturel de vanille douce protège vos cheveux et leur apporte douceur et souplesse.

\section{$<(8)$ Casino, Douche>}

[Sur les parfums (1) à (4) les mots qui varient ont été soulignés.]

\section{(1) DOUCHE CACAO DELICAT}

Avec le lait de douche Casino Cacao délicat, transformez votre douche en un véritable moment gourmand. De $\mathrm{pH}$ neutre pour la peau, sa formule enrichie en provitamine B5 laisse votre peau souple, douce et hydratée. Sa mousse onctueuse et sa texture crémeuse vous procurent une agréable sensation de bien-être en enveloppant votre corps d'un parfum suave et gourmand.

\section{(2) LAIT DE DOUCHE PECHE VELOUTEE}

Avec le lait de douche Casino Pêche veloutée, transformez votre douche en un véritable moment de plaisir. De $\mathrm{pH}$ neutre pour la peau, sa formule enrichie en provitamine B5 et en extrait de pêche laisse votre peau souple, douce et hydratée. Sa mousse onctueuse et sa texture crémeuse vous procurent une agréable sensation de bien-être en enveloppant votre corps d'un parfum fondant et fruité.

\section{(3) LAIT DE DOUCHE DELICIEUSE COCO}

Avec le lait de douche Casino Délicieuse coco, transformez votre douche en un véritable moment de détente exotique. De pH neutre pour la peau, sa formule enrichie en provitamine B5 et en extrait de noix de coco laisse votre peau souple, douce et hydratée. Sa mousse onctueuse et sa texture crémeuse vous procurent une agréable sensation de bien-être en enveloppant votre corps d'un parfum doux et gourmand.

\section{(4) LAIT DE DOUCHE VANILLE GOURMANDE}

Avec le lait de douche Casino Vanille gourmande, transformez votre douche en un véritable moment de plaisir. De $\mathrm{pH}$ neutre pour la peau, sa formule enrichie en provitamine B5 et en extrait de vanille laisse votre peau souple, douce 
et hydratée. Sa mousse onctueuse et sa texture crémeuse vous procurent une agréable sensation de bien-être en enveloppant votre corps d'un parfum suave et gourmand.

\section{(5) LAIT DE DOUCHE AMANDE DOUCE}

Conjuguez plaisir et soin avec le lait de douche Amande douce Casino. De pH neutre pour la peau, sa formule enrichie en provitamine B5 et en extrait d'amande douce offre à votre peau beaucoup de douceur, la laissant veloutée et hydratée. Sa texture crémeuse et sa mousse onctueuse enveloppent votre corps d'un parfum doux et subtil.

\section{(6) DOUCHE CREME SURGRAS}

De pH neutre pour la peau et testée sous contrôle dermatologique, la douche crème dermoprotectrice surgras Casino a été spécialement conçue pour les peaux sensibles. Sa formule contenant un extrait de calla et du beurre de karité vous aide à conserver une peau belle et saine et préserve son équilibre naturel. Sa mousse onctueuse et délicatement parfumée transforme votre douche en un véritable moment de plaisir et vous enveloppe de douceur.

<(9) Petit Marseillais, Douche>

(1) [recto] Beurre de karité \& Acacia de Tunisie - Douche Crème hydratation intense

[verso] Pour prendre soin de votre peau, Le Petit Marseillais s'est inspiré des secrets de beauté des femmes du Sud et vous propose ses Douches Recettes Méditerranéennes.

Les secrets des ingrédients méditerranéens...

Extrait de la noix, le beurre de karité est reconnu pour ses vertus hydratantes et nourrissantes. L'acacia de Tunisie révèle un parfum délicat et sucré.

...pour une peau intensément hydratée

La Douche Crème Hydratation Intense lave en douceur, hydrate immédiatement et durablement les peaux sèches et déshydratées.

...et des instants de pur plaisir

Sous la douche, sa texture crème se transforme en une mousse onctueuse et généreuse. Cette douche se rince en un clin d'œil et dépose sur votre peau un parfum enveloppant et gourmand, comme une évasion sous le soleil de Tunisie.

Plus qu'un gel douche, un véritable soin au quotidien : votre peau est douce, nourrie et hydratée.

\section{(2) [recto] Huile d'argan \& Fleur d'oranger du Maroc - Douche Huile nourrissante}

[verso] Pour prendre soin de votre peau, Le Petit Marseillais s'est inspiré de Recettes Méditerranéennes aux ingrédients authentiques et vous propose une gamme complète de douches, adaptées à chacun de vos besoins.

Les secrets des ingrédients méditerranéens...

L'huile d'Argan et la fleur d'Oranger sont deux ingrédients majeurs des rituels de beauté des femmes d'Afrique du Nord. Rare et précieuse, l'huile d'argan est utilisée pour ses vertus nourrissantes. La fleur d'oranger du Maroc, au parfum raffiné, est connue pour ses propriétés apaisantes.

\section{...pour une peau nourrie et satinée}

La Douche Huile Nourrissante lave en douceur, hydrate, et nourrit la peau en un seul geste.

...et des instants de pur plaisir

Sous la douche, sa texture fluide se transforme en mousse généreuse adaptée aussi au rasage. Non grasse, cette douche se rince en un clin d'œil et dépose sur votre peau un parfum sensuel et chaleureux, comme une évasion sous le soleil marocain.

Plus qu'une douche, un véritable soin au quotidien : votre peau est douce, souple et protégée.

\section{(3) [recto] Lait de jasmin \& Hibiscus d'Égypte - Douche au Lait hydratante}

[verso] Pour prendre soin de votre peau, Le Petit Marseillais s'est inspiré des secrets de beauté des femmes du Sud et vous propose ses Douches Recettes Méditerranéennes.

Les secrets des ingrédients méditerranéens...

Le lait de jasmin est utilisé pour ses vertus hydratantes. Fleur emblématique des jardins [é]gyptiens, l'hibiscus délivre un parfum floral délicat. ...pour une peau douce et hydratée

La douche au lait hydratante lave en douceur, adoucit et assouplit la peau.

...et des instants de pur plaisir 
Sous la douche, sa texture lactée se transforme en une mousse onctueuse. Facile à rincer, cette douche enveloppe votre peau d'un parfum fleuri, comme une invitation à découvrir les mystères de l'Égypte.

Plus qu'un gel douche, un véritable soin au quotidien : votre peau est douce, souple et hydratée.

(4) [recto] Lait d'amandes \& Miel du Péloponnèse - Douche au Lait hydratante

[verso] Pour prendre soin de votre peau, Le Petit Marseillais s'est inspiré de Recettes Méditerranéennes aux ingrédients authentiques et vous propose une gamme complète de douches, adaptées à chacun de vos besoins.

Les secrets des ingrédients méditerranéens...

L'amande et le miel sont deux ingrédients majeurs des rituels de beauté des femmes du Sud. Précieux élixir, le miel du Péloponnèse est apprécié pour ses vertus adoucissantes. Le lait d'amandes est utilisé pour ses bienfaits nourrissants.

...pour une peau durablement hydratée

La Douche au Lait Hydratante lave en douceur, hydrate intensément et nourrit la peau en un seul geste. ...et des instants de pur plaisir

Sous la douche, sa texture lactée se transforme en une mousse onctueuse, adaptée aussi au rasage. Facile à rincer, cette douche enveloppe votre peau d'un parfum doux et gourmand, comme une invitation aux délices de la Grèce.

Plus qu'une douche, un véritable soin au quotidien : votre peau est douce, souple et protégée.

$<(11)$ Super Croix, Lessive>

(1) Super Croix Secrets d'Ailleurs

Découvrez Super Croix Secrets d'Ailleurs Maroc : fleur d'oranger et lait d'amande douce.

En plus de vous assurer une propreté parfaite, Super Croix Secrets d'Ailleurs est allé puiser au cœur de la fleur d'oranger* un parfum ensoleillé et dépaysant pour une expérience parfumée unique. Super Croix Secrets d'Ailleurs a également sélectionné au cœur des traditions du monde un ingrédient authentique, le lait d'amande douce, reconnu pour sa grande douceur.

Ainsi votre linge vous offre un vrai moment de plaisir et d'évasion à chaque lavage.

Les secrets de la fleur d'oranger

Très odorante, la fleur d'oranger est à la fois stimulante et apaisante. Son parfum exquis vous emporte instantanément dans un pays de couleurs et de soleil, de gaieté et de générosité.

Les secrets du lait d'amande douce

Super Croix Secrets d'Ailleurs vous dévoile les secrets de l'amande douce, un ingrédient authentique et naturel, très prisé en cosmétique. L'amande douce provient de l'amandier, originaire d'Asie centrale et cultivé dans les pays méditerranéens. Réputé pour ses propriétés adoucissantes et hydratantes, il est particulièrement recommandé pour les peaux sèches.

Évadez-vous le temps d'un lavage avec Super Croix Secrets d'Ailleurs !

Retrouvez tout le plaisir des lessives Super Croix avec les gammes :

Bora-Bora : Fleur de Monoï* et Lait d'Aloe

Plongez dans l'univers lointain et magique de Bora-Bora. Découvrez le parfum* exotique et raffiné de la Fleur de Monoï* allié à la grande douceur du Lait d'Aloe. La lessive Super Croix Secrets d'Ailleurs Bora-Bora Fleurs de Monoï* et Lait d'Aloe existe en formats liquide, poudre et tablettes.

Madagascar : Fleur d'Orchidée* et Lait de Palme

Retrouvez l'atmosphère tropicale et envoûtante de Madagascar. Essayez le parfum* élégant et voluptueux de la Fleur d'Orchidée* allié à l'irrésistible douceur du Lait de Palme. La lessive Super Croix Secrets d'Ailleurs Fleur d'Orchidée* et Lait de Palme existe en formats liquide et tablettes.

Vietnam : Fleur de Lotus* et Lait de Bambou

[Ici, contradiction : l'image montre du bambou, mais il est en fait remplacé par du «lait d'aloe », v. emballage cidessous.]

Découvrez l'atmosphère fascinante et dépaysante du Vietnam. Essayez le parfum* doux et délicat de la Fleur de Lotus* allié à la grande douceur du Lait d'Aloe. La lessive Super Croix Secrets d'Ailleurs Fleur de Lotus* et Lait d'Aloe existe en format liquide.

*parfum de synthèse

(2) Super Croix Secrets d'Ailleurs

Découvrez Super Croix Secrets d'Ailleurs Lotus du Japon* et lait d'aloe 
En plus de vous assurer une propreté parfaite Super Croix Secrets d'Ailleurs est allé puiser au cœur du Lotus du Japon* un parfum* délicat et rare pour une expérience parfumée unique. Super Croix Secrets d'Ailleurs a également sélectionné au cœur des traditions du monde les essences naturelles les plus pures et un ingrédient authentique, le Lait d'Aloe, reconnu pour sa grande douceur.

Ainsi votre linge vous offre un vrai moment de plaisir et d'évasion à chaque lavage.

Les secrets du Lotus du Japon

$\mathrm{Au}$ cœur du Japon, se développe une fleur aquatique extraordinaire : le Lotus. Symbole de pureté, cette fleur très recherchée est également considérée comme sacrée en Asie. Le Lotus diffuse un parfum doux et agréable et vous procure d'incroyables sensations de bien-être.

Les secrets du Lait d'Aloe

Super Croix Secrets d'Ailleurs vous dévoile les secrets de l'Aloe, un ingrédient fabuleux, très prisé en cosmétique. L'Aloe est une plante magique, qui a la capacité de stocker l'eau dans ses feuilles, ce qui lui confère de nombreux bienfaits. Outre des actions apaisantes, l'Aloe ou le Lait d'Aloe, possèdent ainsi des propriétés adoucissantes et purifiantes.

Évadez-vous le temps d'un lavage avec Super Croix Secrets d'Ailleurs !

[+ encadrés faisant référence aux autres parfums de la gamme, v. emballage (1).] 